\title{
Incidental focal intracranial computed tomographic findings
}

\author{
LEON A WEISBERG
}

From the Department of Neurology and Psychiatry, Tulane School of Medicine and the Tulane Department of Neurology, The Charity Hospital, New Orleans, USA

SUMMARY Of 3000 consecutive computed tomography (CT) head scans there were 28 adult patients who had a focal intracranial lesion, who presented with nonspecific symptoms, and who had normal initial neurological examination. These lesions included cerebral infarction (8), focal atrophy (5), intracerebral haematoma (3), chronic subdural haematoma (3), focal calcification (3) and intracranial neoplasm (6). Neurological consultation was obtained after the CT in all cases. Angiography was subsequently performed in 16 , and eight patients underwent surgery. In no case was there evidence that clinical outcome was improved because of the early CT diagnosis.

Computed tomography (CT) has decreased the need for other neurodiagnostic studies. Because of its rapid acceptance as an imaging technique, utilisation and cost benefit analyses have been limited. Following its introduction, the waiting time for CT was prolonged. This encouraged more judicious use, and head CT scans were usually ordered only by physicians with training in neurodiagnosis and neurological disease. With increasing availability, scans are sometimes ordered by physicians who have no formal training in neurodiagnosis. In certain cases, the patients have no prior neurological consultation, and the scans are performed with poor indication. The purpose of this report is to analyse the clinical findings and outcome in 28 patients who had an incidental focal lesion detected by CT which required much time and expense to study and evaluate with little ultimate benefit.

\section{Methods and material}

Three thousand consecutive adult patients who had CT head scans were retrospectively analysed. Twenty-eight who had a focal abnormality were reported as having had a normal initial neurological examination, but none had had a prior neurological or neurosurgical consultation. Patients with systemic carcinoma, for example of lung or breast, in whom CT was performed as a staging procedure were excluded. The clinic and hospital records were analysed for the following information, (1) symptomatology necessitat-

Address for reprint requests: Leon A Weisberg, MD, Department of Neurology and Psychiatry, Tulane Medical Center, 1415 Tulane Avenue, New Orleans, LA 70112, USA.

Received 30 January 1982. Accepted 9 April 1982 ing hospitalisation, (2) neurodiagnostic studies performed prior to CT, (3) studies performed as a consequence of the abnormal CT, (4) the results of the neurological consultation, (5) subsequent hospital stay (prolonging hospital course) and treatment which resulted from the CT finding.

\section{Results}

The symptoms for which the scan was performed is listed in table 1 . The lesions visualised by CT are listed in table 2. Eight patients showed CT findings consistent with a cerebral infarction. These patient's

Table 1 Neurological symptomatology in 28 patients with incidental CT findings

\begin{tabular}{lr}
\hline $\begin{array}{l}\text { Headache only } \\
\text { band-like 5 }\end{array}$ & 8 \\
throbbing 3 & 6 \\
"rule out intracranial pathology" & 4 \\
Light-headedness & 3 \\
Diffuse paraesthesias & 2 \\
Fainting spells & 2 \\
General weakness & 2 \\
Cachexia and weight loss & 1 \\
Visual blurring & \\
\hline
\end{tabular}

Table 2 Diagnoses established by CT scan findings

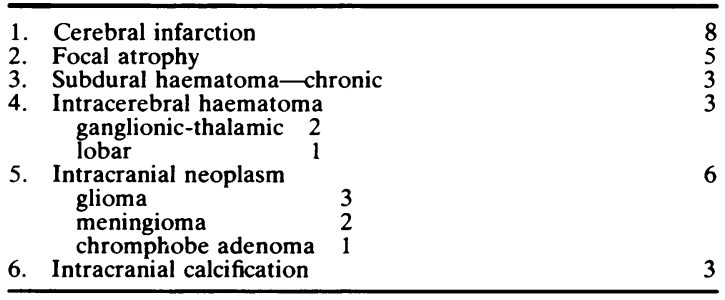


ages ranged from 44 to 56 years. Initial history was reported as negative for cerebrovascular symptomatology; neurological examination was reported as normal and no carotid bruits were heard. These patients were initially hospitalised for symptoms including light-headedness, chest pain, palpitation, shortness of breath; however, none was found to have evidence of cardiac arrhythmias, myocardial infarction, cardiomyopathies or valvular heart disease. Four patients had systemic arterial hypertension and four others were normotensive. Prior to CT all patients had electroencephalograms (EEG), five of which were normal and three showed focal slow wave pattern. Only two patients had isotope brain scans; both had normal dynamic and static studies.

Following demonstration of CT abnormality, neurological consultation was obtained in all cases. Retrospective history indicated symptoms consistent with prior cerebral hemispheric (carotid territory) transient ischaemic attacks in all (8) patients; four of them also described episodes of focal neurological deficit lasting 24 to 72 hours. No patient had a transient ischaemic attack in the 3 months preceding CT. The consultant found mild focal neurological deficit in five patients. In three patients, CT showed postcontrast grey matter enhancement and angiography was performed to exclude a neoplasm or vascular malformation. One of three patients developed a hemiparesis-hemisensory deficit immediately following angiography and this persisted for 11 days. The performance of angiography in these patients prolonged hospitalisation by 6 to 14 days. Five other patients did not have angiography; hospitalisation was prolonged 4 to 8 days to obtain isotope brain scan, CSF analysis, noninvasive carotid flow studies, and cardiology evaluation (including echocardiogram and 24 hours Holter cardiac monitor to exclude potential embolic source).

On the basis of the CT diagnosis of cerebral infarction, all patients were treated with aspirin and dipyridamole. Follow-up CT was performed 2 to 6 weeks later in five patients; this showed no interval change in two patients with hypodense nonenhancing lesions and resolution of enhancement in three others. These eight patients were followed for 3 to 13 months by the neurologist and remained clinically stable and had no further transient ischaemic episodes.

Three patients had CT evidence of intracerebral haematoma, but no mention was made in the initial history of a stroke syndrome. They were men (age $52,57,59$ years); all had systemic arterial hypertension and were taking multiple antihypertensive medications. The purpose of the hospitalisation was to improve the blood pressure control as their physicians believed they were noncompliant. EEG and isotope scan were normal in all cases. The history subsequently obtained by the neurologist indicated a prior episode which was characterised by sudden onset of severe but evanescent headache followed by focal weakness or numbness lasting 3 to 4 days. This had occurred 2 to 4 weeks prior to hospitalisation. The neurologist reported that two patients had focal neurological deficit. The CSF was clear. A haematoma in the basal ganglia was believed to be of hypertensive aetiology; angiogram was not performed but an evaluation for a coagulation disorder was undertaken. Two other patients with lobar haematomas had angiography to exclude an underlying aetiology (angioma, aneurysm or neoplasm). In these three cases, hospitalisation was prolonged 6 to 12 days following CT; however medical treatment was not altered by this finding. In one case, followup CT showed resolution of the haematoma three months later.

Five patients had CT evidence of focal cerebral hemispheric atropy. The purpose of CT was to define an aetiology for diffuse paraesthesiae and light-headedness in two patients; three others had no neurological symptoms and indication for CT was to "rule out intracranial pathology". These patients were being evaluated for diabetes, abdominal pain, urological symptoms and neck pain. EEG had been performed prior to CT in one case; it showed slight focal slowing. Following CT, EEG was performed in four others, it showed focal slowing in two and was normal in two. Isotope scan was negative in all five cases. Neurological consultation showed unilateral pronator drift in one case, homonymous hemianopsia in one and was normal in three. Two patients had a prior history of head trauma accompanied by loss of consciousness. No patient underwent angiography. Hospitalisation was prolonged for 3 days after the CT to obtain neurological consultation, EEG and isotope scan. None of the patients had seizures and none have developed seizures within follow-up extending for 12 to 24 months.

Three patients had CT scan evidence of a unilateral chronic subdural haematoma. In two cases, there was evidence of mass effect but this was absent in one other. These patients had been hospitalised because of systemic medical disease; but CT was performed for headache only. The headache was located in the occipital region, was aching and bandlike in nature, did not awaken the patient and was not associated with vomiting. Prior to CT, isotope scan was negative in all, and EEG was normal in two and diffusely slow in one. Following CT demonstration of subdural haematoma, neurological consultant found evidence of pronator drift and Babinski sign in two and no abnormality in one. Angiography confirmed the presence of the haematoma in all 
cases. One patient who had no abnormal neurological finding and CT evidence of subdural haematoma without mass effect did not have surgery; he remains asymptomatic 14 months later. The hospital course was prolonged 6 days for angiography, neurological, and neurosurgical consultation. He has had two further CT scans which show no change in the lesion. Two patients had surgery with drainage of 50 to $75 \mathrm{ml}$ of liquified proteinaceous fluid with disappearance of the subdural mass on CT. One patient was neurologically normal, but one patient developed non-haemorrhage infarction with residual hemiparesis and hemisensory deficit. Hospitalisation was prolonged 16 to 20 days in these two cases.

In three patients, CT showed evidence of calcification; this was in the frontal-parietal, medial temporal and basal ganglia regions. In these three patients, the indication for the CT was to "rule out intracranial pathology" as hospitalisation was necessitated for an acute medical problem. EEG showed a focal spike pattern in two and was normal in one; isotope scan was normal in three. No calcium or phosphate abnormality was discovered. In two patients with an EEG spike focus, angiography was performed to exclude an underlying mass or vascular malformation. For this, hospitalisation was prolonged 6 to 11 days. Follow-up in these three patients has extended for 9,12 and 26 months; neither seizures nor interval CT change has occurred.

Three patients had CT findings consistent with a primary intracranial malignant neoplasm. One patient was evaluated for syncope; two others had dizziness and head pain with weight loss and anorexia. Prior to CT, EEG showed mild degree focal slowing in two and was normal in one; isotope scan was negative in all. The neurologist's examination showed focal deficit in two patients; the other patient had obvious episodes of partial complex seizures. Angiography was performed in all and it showed an avascular mass. All patients had surgery and had histological evidence of a intracranial glioma. Following surgery neurological deficit worsened in two; major motor seizures developed in all within 2 weeks of operation. All received brain irradiation. Hospitalisation was prolonged for 5 to 8 weeks by subsequent diagnostic and therapeutic intervention. These patients later required reoperation for tumour recurrence within 5 months of initial treatment. Two patients had CT findings consistent with meningioma. The parietal convexity lesion was $2.8 \mathrm{~cm}$ in diameter and there was slight evidence of mass effect without hydrocephalus; the tentorial meningioma was $1.2 \mathrm{~cm}$ in diameter without associated hydrocephalus. Symptoms included headache and dizziness. Prior to CT, EEG was normal in both cases; isotope scan was positive in the paretial and negative in the tentorial tumour. Neurological examination performed by both neurology and neurosurgical consultants were negative. Angiographic findings were consistent of meningioma; this diagnosis was confirmed surgically. Hospitalisation was prolonged by 16 to 26 days by surgery. Nine months post-surgery the patient with the parietal meningioma developed persistent focal seizures with no CT evidence of tumour recurrence; and the patient with the tentorial meningioma had a severe gait ataxia.

One patient had CT evidence of an intrasellar non-calcified enhancing lsion with suprasellar extension; however he had no endocrine or visual symptoms. This patient was hospitalised because of headache, lightheadedness and inabiity to concentrate which had developed after mild head trauma. Following CT, tangent screen visual field examination was normal. Angiography confirmed the CT findings and showed no evidence of aneurysm. $\mathrm{Bi}$ frontal craniotomy was performed with removal of a chromophobe adenoma. Hospitalisation was for 6 weeks. Following completion of radiotherapy, visual fields showed superior bitemporal quadrantopsia to $5 \mathrm{~mm}$ red test object.

\section{Discussion}

In the initial period following the introduction of CT, there was a waiting period of up to 2 months for CT. Because of this limited access, physicians with training in neurological disorders acted as a triage to determine the need for CT. With the dramatic increase in the number of scanners installed and a decrease in the waiting time for CT, this triage has been abandoned; physicians without specialised training in neurological disorders or neurodiagnostic techniques routinely order CT, and in patient's with questionable indications. ${ }^{12}$ In this study, 28 patients who had a focal CT lesion did not have a prior neurological consultation and in no case is there evidence that outcome was improved by early CT diagnosis. Many more CT scans are ordered than should be. From this study it was not possible to assess the incidence because many scans were ordered from several different patient sources. In certain institutions very careful screening for $\mathrm{CT}$ was performed but in others no screening was possible.

This trend toward CT without initial neurological consultation was not related to lack of availability of neurologists or neurosurgeons, but it appears that CT was being used as a screening neurological procedure. This approach generates several types of problems; (1) a negative CT study does not exclude 
the presence of neurological disease, for example meningitis and subarachnoid haemorrhage are diagnosed by lumbar puncture and CSF analysis; (2) overreliance on CT diagnostic accuracy especially if the clinical findings suggest an alternative diagnosis; for example, when the CT findings show cerebral atrophy but clinical findings suggest a functional affective disorder; ${ }^{3}$ (3) aggressive management of incidental lesions.

Necropsy studies have reported that $30 \%$ of normal patients have incidental pituitary adenomas. ${ }^{4}$ These lesions are usually small; however certain larger lesions which would have been expected to cause endocrine or visual deficit have also been reported. Incidental meningiomas have also been reported. ${ }^{5}$ With the increased diagnostic sensitivity of CT, certain of these incidental tumours are now detected; and this presents a therapeutic dilemma. The anaesthetic and surgical risks combined with the unknown benefit associated with early removal in an asymptomatic patient makes this an uncertain decision. Prior studies have reported the increased incidence of low-grade (Grade I) gliomas detected in the post-CT era; however, early diagnosis has not had a positive effect on outcome. ${ }^{6}$ In three patients who had CT and operative findings of glioma (Grade I, 2 cases; Grade II, 1 case), the outcome was poor with early recurrence. In the three other patients with intracranial neoplasms, outcome was not improved by early diagnosis.

In patients with symptoms of cerebrovascular disease, CT is completely accurate in excluding clinically symptomatic intracerebral haematoma and is quite reliable in detecting cerebral infarction. In two prior studies ${ }^{78}$ the CT diagnosis of an intracranial haematoma was clinically unsuspected, similar to the patients in this series. Abnormal CT findings in cerebral infarction consisting of grey matter enhancement has been reported in rare instances of patients with transient ischaemic attacks.' Careful neurological history in the eight patients with CT evidence of cerebral infarction or ischaemia documented prior vascular episodes which were previously undetected by the non-neurologist. The abnormal CT findings may be important in assessing the need for anticoagulation, angiography or carotid endarterectomy.

The natural history of subdural haematoma is not definitely known. ${ }^{10}{ }^{11}$ It is assumed that it causes neurological disturbances when mass effect develops; however two patients were asymptomatic even though mass effect was present and focal findings were present. In one patient, there has been no change in CT appearance or clinical findings 14 months later with no surgery or corticosteroid. In this case, since the hypodense lesion effaced the cortical sulcal spaces with normal sized and positioned ventricles, it is possible that this represents a subdural haematoma and not a subdural hygroma or focal atrophy.

It has been reported that almost one-quarter of patients who are admitted to medical services in community hospitals have major neurological problems. ${ }^{1}$ If these patients are not carefully assessed clinically with critical evaluation of the need for CT, two potentially dangerous trends may develop. Firstly, unnecessary diagnostic and therapeutic intervention may be carried out in asymptomatic patients with incidental unrelated lesions. Secondly, a negative CT scan result may then be accepted as evidence that neurological disease does not exist; and consultation from a physician with training in neurodiagnosis is not subsequently obtained.

\section{References}

' Levitt LP. Neurological education on non-neurologists. Neurology (Minneap) 1979;29:1515-16.

${ }^{2}$ Oldendorf WH. The quest for an image of brain: a brief historical and technical review of brain imaging techniques. Neurology (Minneap) 1978;28:517-23.

${ }^{3}$ Wells CE, Duncan GW. Danger of over-reliance on computerized cranial tomography. Am J Psychiatry 1977;134:811-3.

4 Costello RT. Subclinical adenoma of the pituitary gland. Am J Pathol 1936;12:205-20.

${ }^{5}$ Wood MW, White RJ, Kernohan JW. One hundred intracranial meningiomas found incidentally at necropsy. J Neuropathol Exp Neurol 1.957;16:337-43.

- Baker HL. CT and neuroradiology: a fortunate primary union. Am J Roentgenology 1976;127:101-7.

' Rudick RA. Asymptomatic intracerebral hematoma as an incidental finding. Arch Neurol 1981;38:396.

${ }^{8}$ Greenberg JD, Skubick DL. Unexpected brain hemorrhages and the value of computerized tomography. Comput Tomogr 1977;1:349-57.

${ }^{9}$ Kinkel WP, Jacobs L, Kinkel PR. Gray matter enhancement: a computerized tomographic sign of cerebral hypoxia. Neurology (Minneap) 1980;30: 810-19.

${ }^{10}$ Bender MB, Christoff N. Nonsurgical treatment of subdural hematoma. Arch Neurol 1974;31:73-7.

"Lusins J, Jaffe R, Bender MB. Unoperated subdural hematoma. J Neurosurg 1976;44:601-6. 